\title{
Resilient meerkats
}

Meerkats (Suricata suricatta) living in the Kalahari Desert must cope with extreme variations in temperature and rainfall throughout the year. Writing in Science, Maria Paniw and colleagues report that these variations alter the animals' body mass, and that body-mass changes have different effects on meerkat populations depending on when they happen (M. Paniw et al. Science 363, 631-635; 2019).

For example, low rainfall just before the breeding season starts leads to food scarcity, low body mass, low reproductive success and an increased risk of population extinction. But a warm environment during the non-breeding season can increase body mass and lead to more efficient reproduction, compensating for previous losses in population size.

The findings are of broad interest because species living in extreme seasonal environments, such as meerkats, give us a glimpse of the ecological effects of future changes in Earth's climate. Joana 0sório

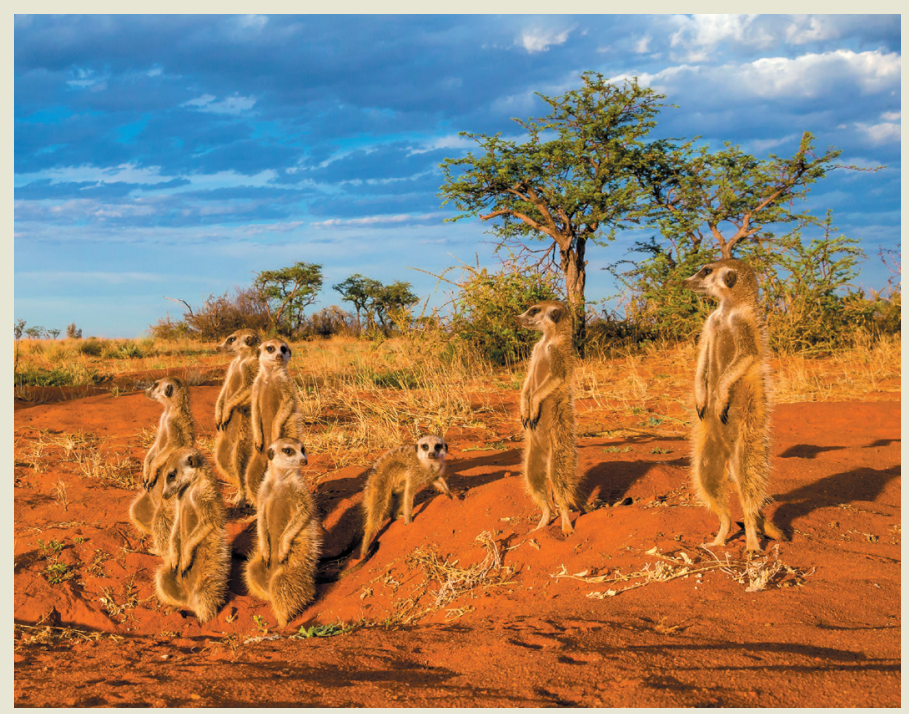

\section{Hosts combat Ebola} using protein disguise

\begin{abstract}
Infection by Ebola virus can be fatal. The discovery of a human protein that mimics one type of Ebola protein and binds to another to suppress viral RNA production might aid the development of clinical treatments for the disease.
\end{abstract}

\section{SEIYA YAMAYOSHI \& YOSHIHIRO KAWAOKA}

$\mathrm{T}$ The natural hosts for the Ebola virus are thought to be bats. However, this RNA virus can also infect humans, and there have been numerous reported outbreaks of the viral infection originating in African countries over the past 40 years ${ }^{1}$. The largest such outin 28,616 suspected cases and 11,310 deaths, mainly in Guinea, Liberia and Sierra Leone (go.nature.com/2qtbj6i). The fatality rate can be high: for example, an outbreak that began in 2018 in the Democratic Republic of the Congo has so far resulted in 685 cases of infection and 419 deaths, a fatality rate of approximately $60 \%$ (go.nature.com/2qtdirv).

Ebola infection begins with fever, muscle pain and headache, followed by vomiting, diarrhoea, rash and symptoms of impaired kidney and liver function. Basic supportive care for those infected, such as treatment to combat dehydration, can help to prevent it being fatal ${ }^{2}$. However, in addition to managing symptoms, there is a need to develop other approaches that prevent or treat the disease, such as vaccines, antiviral therapies or antibody treatments $\mathrm{s}^{3-6}$. Although there have been some clinical trials, no drugs or vaccines have yet been approved for clinical use. And because it can't break was between 2013 and 2016, and resulted be predicted where the next Ebola outbreak will occur, it is difficult to identify those most at risk of infection, and so plan a vaccination strategy. Writing in Cell, Batra et al. ${ }^{7}$ report their investigation of natural host defences against the Ebola virus. Their identification of a human protein that can affect the success of viral replication might open new avenues of research into antiviral treatments.

Batra and colleagues expressed tagged versions of Ebola proteins individually in human cells grown in vitro, and used co-immunoprecipitation and mass spectrometry techniques to identify human proteins that interacted with viral proteins. They used this information to generate a map of the network of such interactions - termed an interactome map. The authors found 194 interactions between host and viral proteins, one of which was between the human protein RBBP6 - a type of enzyme called a ubiquitin ligase - and an Ebola protein called VP30. Various Ebola proteins, including VP30, function in the viral polymerase protein complex, which makes a Synthesis of Ebola virus RNA

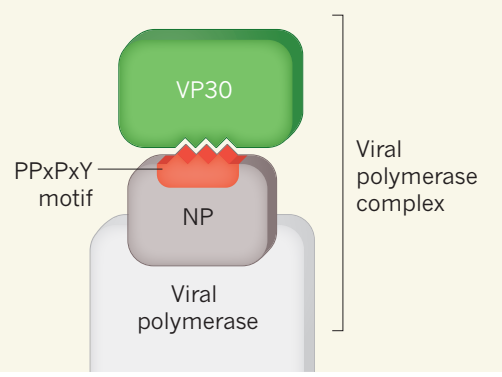

b Decrease in synthesis of

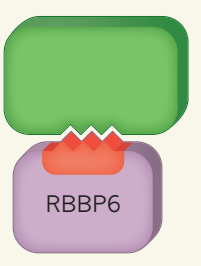
Ebola virus RNA

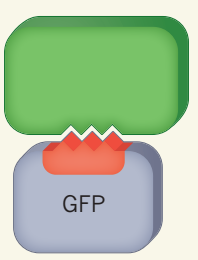

Figure 1 Protein interactions and a host defence response to Ebola virus infection. a, When the Ebola virus infects human cells, the virally encoded proteins VP30 and NP interact ${ }^{8}$, helping to boost the synthesis of Ebola virus RNA. NP binds to the viral polymerase enzyme, and is part of the viral polymerase protein complex that makes viral RNA. Batra et al. ${ }^{7}$ report that a motif of amino-acid sequences termed PPxPxY has a key role in the interaction between VP30 and NP. b, To uncover possible host defences against infection by Ebola virus, the authors used human cells grown in vitro to test for host proteins that could bind to proteins encoded by the virus. They report that the human protein RBBP6 has a PPxPxY motif through which it binds to VP30. Because this binding inhibits the interaction of NP with VP30, it helps to limit the synthesis of Ebola virus RNA in human cells grown in vitro. The authors report that if a peptide that contains the PPxPxY motif is fused to green fluorescent protein (GFP), the resulting chimaeric protein also inhibits this viral RNA synthesis in human cells grown in vitro. 